\title{
RESEARCHES OF THE PROPERTIES OF MATERIALS USED FOR PROTECTION FROM NEUTRON RADIATION USING BONNER SPHERE SPECTROMETER WITH A ${ }^{3} \mathrm{He}-\mathrm{COUNTER}$
}

\author{
V.B. Ivanskyi ${ }^{1}$, O.N. Letuchyy ${ }^{1}$, A.N. Orobinskyi ${ }^{1}$, V.E. Ostrovskykh ${ }^{2}$, H.V. Siroko ${ }^{1}$ \\ ${ }^{I}$ National Scientific Centre "Institute of Metrology» \\ 42, Mironosickaya Str., Kharkov, 61002, Ukraine \\ ${ }^{2}$ Industrial and commercial private enterprise "OST» \\ 82, Ak. Pavlova Str., Kharkov, 61038, Ukraine \\ E-mail:orobin61@gmail.com \\ Received 8 September, 2017; accepted 7 October, 2017
}

The energy and pulse height spectrums after passage of neutron radiation through samples of materials most often used to protect against neutron radiation (paraffin, paraffin with boric acid, paraffin with boric acid and lead, polyethylene, polyethylene with boron and boron carbide) are measured using a PuBe neutron source using a NEMUS spectrometer on Bonner spheres with ${ }^{3} \mathrm{He}$-counter. The values of linear attenuation coefficients and neutron relaxation lengths in these materials, neutron spectra for these materials are given. The percentage of neutrons in the energy subbands is determined according to the radiation weighting factor. The neutron flux density, the ambient dose equivalent power, the absorbed dose rate in the neutron radiation air, the transition coefficients from the neutron fluence to the ambient dose equivalent and average neutron radiation energy are measured. Calculation of energy spectrum, basic characteristics of neutron radiation and extended uncertainties is performed by the MAXED (maximum entropy and deconvolution), applying the Bayes theorem, method using the UMG_3.3 software.

KEY WORDS: protection from neutron radiation, linear neutron attenuation coefficient, neutron relaxation length in the material, neutron radiation spectrum, neutron flux density, ambient dose rate equivalent, absorbed dose rate, NEMUS spectrometer on Bonner spheres, ${ }^{3} \mathrm{He}$ neutron counter, Bayes theorem, MAXED method.

\section{ДОСЛІДЖЕННЯ ВЛАСТИВОСТЕЙ МАТЕРІАЛІВ, ЯКІ ЗАСТОСОВУЮТЬСЯ ДЛЯ ЗАХИСТУ ВІД НЕЙТРОННОГО ВИПРОМІНЕННЯ, ЗА ДОПОМОГОЮ СПЕКТРОМЕТРА

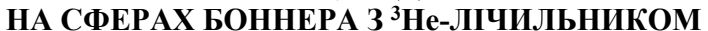 \\ В.Б. Іванський ${ }^{1}$, О.М. Летучий ${ }^{1}$, А.М. Оробінський ${ }^{1}$, В.С. Островських ${ }^{2}$, Г.В. Сіроко ${ }^{1}$ \\ ${ }^{1}$ Наиіональний науковий иентр «Інститут метрологї̈» \\ 42, вул. Мироносиџька, м. Харків, 61002, Україна \\ ${ }^{2}$ Виробниче-комериійне приватне підприємство «ОСТ» \\ 82, вул. Ак. Павлова, м. Харків, 61038, Україна}

Виміряні енергетичні та амплітудно-імпульсні спектри після проходження нейтронного випромінення крізь зразки матеріалів, які найбільш часто застосовуються для захисту від нейтронного випромінення (парафін, парафін з борною кислотою, парафін 3 борною кислотою та свинцем, поліетилен, поліетилен з бором та карбід бору) при використанні РuВе джерела нейтронів за допомогою спектрометра NEMUS на сферах Боннера з ЗНе-лічильником. Наведено значення лінійних коефіцієнтів ослаблення і довжин релаксації нейтронів в цих матеріалах, спектри нейтронів за даними матеріалами. Визначено відсоткове співвідношення нейтронів в піддіапазонах енергій згідно радіаційному зважуючому фактору. Виміряні густина потоку нейтронів, потужність амбієнтного еквівалента дози, потужність поглинутої дози в повітрі нейтронного випромінювання, коефіцієнти переходу від флюенса нейтронного випромінення до еквівалентної дози і середні енергії спектрів. Розрахунок енергетичних спектрів, основних характеристик нейтронного випромінення та розширених невизначеностей виконано методом MAXED (максимальної ентропії та деконволюції), застосовуючи теорему Байєса, за допомогою програмного забезпечення UMG_3.3.

КЛЮЧОВІ СЛОВА: захист від нейтронного випромінення, лінійний коефіцієнт ослаблення нейтронів, довжина релаксації нейтронів у матеріалі, спектр нейтронного випромінювання, густина потоку нейтронів, потужність амбієнтного еквівалента дози, потужність поглинутої дози, спектрометр NEMUS на сферах Боннера, ${ }^{3}$ Не-лічильник нейтронів, теорема Байєса, метод MAXED

\section{ИССЛЕДОВАНИЕ СВОЙСТВ МАТЕРИАЛОВ, ПРИМЕНЯЕМЫХ ДЛЯ ЗАЩИТЫ ОТ НЕЙТРОННОГО ИЗЛУЧЕНИЯ, С ПОМОЩЬЮ СПЕКТРОМЕТРА НА СФЕРАХ БОННЕРА С ${ }^{3}$ Не-СЧЕТЧИКОМ В.Б. Иванский ${ }^{1}$ А.Н. Летучий ${ }^{1}$, А.Н. Оробинский ${ }^{1}$, В.Е. Островских ${ }^{2}$, Г.В. Сироко ${ }^{1}$ ${ }^{1}$ Наииональный научный иентр «Институт метрологии» 42, ул. Мироносичкая, г. Харьков, 61002, Украина \\ ${ }^{2}$ Производственно-коммерческое частное предприятие «ОСТ》 82, ул. Ак. Павлова, г. Харьков, 61038, Украина}

Измерены энергетические и амплитудно-импульсные спектры после прохождения нейтронного излучения через образцы материалов, которые наиболее часто применяются для защиты от нейтронного излучения (парафин, парафин с борной кислотой, парафин с борной кислотой и свинцом, полиэтилен, полиэтилен с бором и карбид бора) при использовании РuВе источника нейтронов с помощью спектрометра NEMUS на сферах Боннера с ${ }^{3} \mathrm{He-счетчиком.} \mathrm{Приведены} \mathrm{значения} \mathrm{линейных}$ коэффициентов ослабления и длин релаксации нейтронов в этих материалах, спектры нейтронов после прохождения излучения через данные образцы. Определено процентное соотношение нейтронов в поддиапазонах энергий согласно радиационному взвешивающему фактору. Измерены плотность потока нейтронов, мощность амбиентного эквивалента дозы, мощность поглощенной дозы в воздухе нейтронного излучения, коэффициенты перехода от флюенса нейтронного 
излучения к амбиентному эквиваленту дозы и средние энергии спектров. Расчет энергетических спектров, основных характеристик нейтронного излучения и расширенных неопределенностей выполнен методом MAXED (максимальной энтропии и деконволюции), применяя теорему Байеса, при помощи программного обеспечения UMG_3.3.

КЛЮЧЕВЫЕ СЛОВА: защита от нейтронного излучения, линейный коэффициент ослабления нейтронов, длина релаксации нейтронов в материале, спектр нейтронного излучения, плотность потока нейтронов, мощность амбиентного эквивалента дозы, мощность поглощенной дозы, спектрометр NEMUS на сферах Боннера, ${ }^{3}$ Не-счетчик нейтронов, теорема Байеса, метод MAXED

Актуальность данной работы состоит в обеспечении защиты от нейтронного излучения при хранении и транспортировании источников нейтронов.

В данной работе проведены исследования свойств материалов, применяемых для защиты от нейтронного излучения, с целью:

- измерения линейного коэффициента ослабления или длины релаксации нейтронов в материале;

- измерения спектров нейтронного излучения;

- определения процентного соотношения нейтронов в поддиапазонах энергий согласно радиационному взвешивающему фактору;

- измерения плотности потока нейтронов (ППН), мощности амбиентного эквивалента дозы (МАЭД), мощности поглощенной дозы (МПД) в воздухе нейтронного излучения, коэффициента перехода от флюенса нейтронного излучения к амбиентному эквиваленту дозы (АЭД) и средней энергии спектра.

Исследования проведены с помощью спектрометра NEMUS на сферах Боннера [1], разработанного в Physikalisch-Technische Bundesanstalt (PTB), Braunschweig, Germany, с пропорциональным ${ }^{3}$ Не-счетчиком нейтронов SP9 производства фирмы «Centronic Ltd», UK.

Расчет спектров выполнен методом MAXED (максимальной энтропии и деконволюции) [2, 3], применяя теорему Байеса [4].

В известной нам литературе [5 - 8] отсутствует информация о спектрах нейтронного излучения РиВе источника, прошедшего через материалы, применяемые для защиты от данного излучения.

\section{МАТЕРИАЛЫ И МЕТОДЫ}

Структурная схема установки для исследования свойств материалов, применяемых для защиты от нейтронного излучения, приведена на рис. 1.

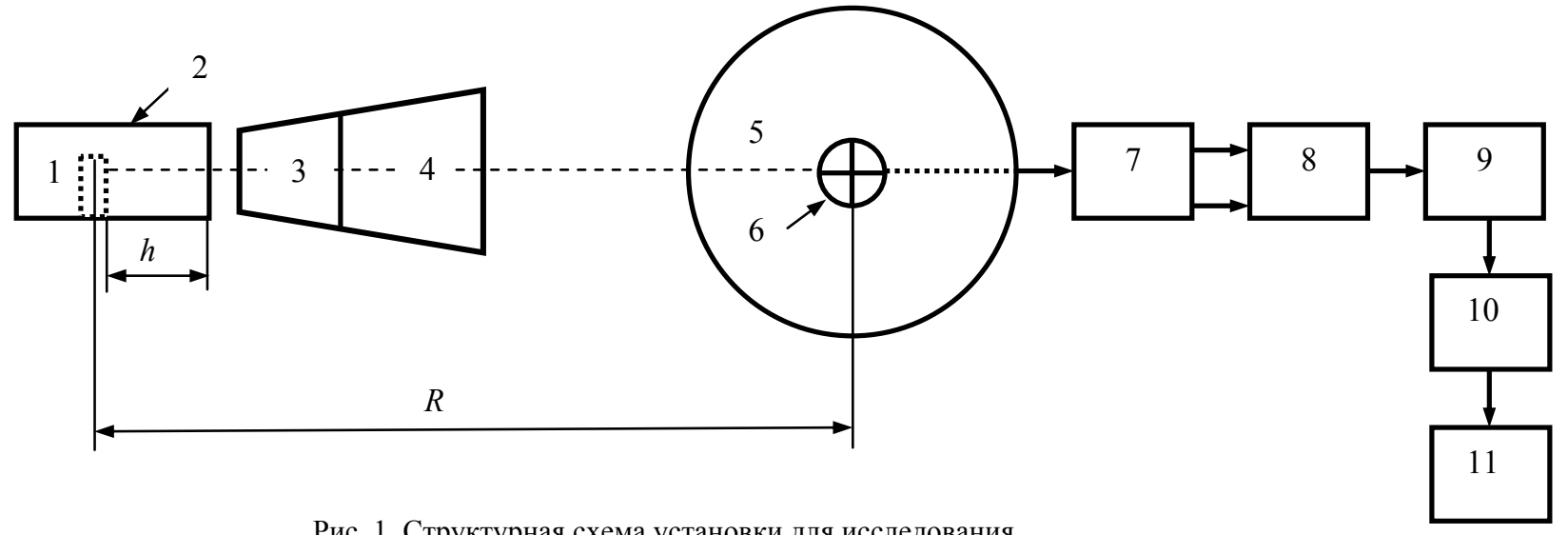

Рис. 1. Структурная схема установки для исследования.

1 - источник нейтронного излучения;

2 - исследуемый образец;

3 - часть теневого конуса из стали;

4 - часть теневого конуса из полиэтилена.

Спектрометр NEMUS на сферах Боннера:

5 - сфера из чистого полиэтилена, применяется 10 сфер: $3 \mathrm{~W} 0(d=3 ") ; 3 \mathrm{~W} 5\left(d=3,5^{\prime \prime}\right) ; 4 \mathrm{~W} 0(d=4 ") ; 4 \mathrm{~W} 5\left(d=4,5^{\prime \prime}\right)$;

5W0 ( $\left.d=5^{\prime \prime}\right) ; 6 \mathrm{W0}(d=6 ") ; 7 \mathrm{W0}(d=7 ") ; 8 \mathrm{W0}(d=8 ") ; 10 \mathrm{W0}(d=10 ")$ и 12W0 $(d=12 ") ; d$ - диаметр сферы;

6 - счетчик SP9 (диаметр - 32 мм) без сферы - 0W0iso (iso - изотропный, 0") (" - дюйм);

7 - универсальный аналоговый спектрометр AIOSAP-02;

8 - аналого-цифровой преобразователь АЦП FAST 7070;

9 - webMATE: Ethernet мультиканальный анализатор (МСA);

10 - NETGEAR FS108P: свич Ethernet;

11 - персональный компьютер с программным обеспечением для измерения спектра нейтронного излучения webMATE [9] совместно с winTMCA32 [10] и для его расчета UMG_3.3 [11, 12];

$h$ - толщина исследуемого образца;

$R=162$ см - расстояние между геометрическими центрами источника и счетчика SP9. 
Образцы исследуемых материалов, которые наиболее часто применяются для защиты от нейтронного излучения [5 - 8], приведены в таблице 1. Форма образцов выбрана в виде параллелепипеда (длина - $\approx 120$ мм, высота - $\approx 80$ мм, ширина - $\approx 60$ мм), в котором размещен источник нейтронного излучения (рис. 1$)$.

Таблица 1.

Образцы исследуемых материалов

\begin{tabular}{|c|c|c|c|c|c|c|}
\hline Образец & $1 \mathrm{~A}$ & $2 \mathrm{~A}$ & $3 \mathrm{~A}$ & $4 \mathrm{~A}$ & $5 \mathrm{~A}$ & $6 \mathrm{~A}$ \\
\hline Материал & парафин & $\begin{array}{c}\text { парафин }+ \\
\text { борная кислота } \\
\left(\mathrm{H}_{3} \mathrm{BO}_{3}-50 \%\right. \\
\text { масс. })\end{array}$ & $\begin{array}{c}\text { парафин }+ \\
\text { борная кислота }+ \\
\text { свинец } \\
\left(\mathrm{H}_{3} \mathrm{BO}_{3}-50 \%,\right. \\
\mathrm{Pb}-5 \% \text { масс.) }\end{array}$ & $\begin{array}{c}\text { полиэтилен } \\
\text { высокого } \\
\text { давления } \\
\text { (ПВД) }\end{array}$ & $\begin{array}{c}\text { ПВД+бор } \\
(\mathrm{B}-3 \% \\
\text { масс.) }\end{array}$ & $\begin{array}{c}\text { карбид } \\
\text { бора }\end{array}$ \\
$\begin{array}{c}\text { Химическая } \\
\text { формула }\end{array}$ & $\begin{array}{c}\mathrm{H}-\left(\mathrm{CH}_{2}\right)_{\mathrm{n}}-\mathrm{H}, \\
18 \leq \mathrm{n} \leq 35\end{array}$ & - & - & $\left.\mathrm{C}_{2} \mathrm{H}_{4}\right)_{\mathrm{n}}$ & - & $\mathrm{B}_{4} \mathrm{C}$ \\
\hline
\end{tabular}

В качестве источника нейтронного излучения выбран РuBe источник типа ИБН-9 (поток нейтронов в телесный угол $4 \pi \mathrm{cp}-B=3,47 \cdot 10^{6} \mathrm{c}^{-1}$; высота - 39 мм, диаметр - 29 мм).

Чтобы учитывать рассеянное нейтронное излучение при измерениях, применяют теневой конус (далее - по тексту конус) (рис. 1) [13, 14].

Плотность потока нейтронов от источника (рис. 1) вычислим по формуле

$$
\varphi=\varphi_{\Sigma}-\varphi_{K},
$$

где $\varphi_{\Sigma}$ - суммарная плотность потока нейтронов, включая рассеянное излучение (без конуса);

$\varphi_{K}-$ плотность потока нейтронов за счет рассеянного излучения (с конусом).

При измерении спектров нейтронного излучения с помощью спектрометра NEMUS на сферах Боннера применяем три конуса, размеры которых приведены в [13, 14].

Измерение спектра выполняется в три этапа [13]:

- измерение амплитудно-импульсных спектров (АИС);

- обработка результатов измерений АИС;

- расчет спектра нейтронного излучения методом MAXED, применяя теорему Байеса.

Скорость счета импульсов от источника нейтронов без учета рассеянного излучения согласно (1) вычислим по формуле

$$
n_{k}=n_{\Sigma k}-n_{K k},
$$

где $n_{\Sigma k}$ и $n_{K k}$ - измеренные скорости счета импульсов без конуса и с конусом.

В для $k$-сферы справедливо выражение [15]:

$$
n_{k}+\varepsilon_{k}=\sum_{i=1}^{n} R_{k i} \cdot \varphi_{i}
$$

где $\varepsilon_{k}=n_{k C}-n_{k}$;

$n_{k C}=\sum_{i=1}^{n} R_{k i} \cdot \varphi_{i} \quad$ и $\quad n_{k C}^{D S}=\sum_{i=1}^{n} R_{k i} \cdot \varphi_{i}^{D S} \quad-$ рассчитанное значение скорости счета импульсов для измеренного ( $\left.\varphi_{i}\right)$ и начального ( $\varphi_{i}^{D S}$, Default Spectrum - DS) спектра $k$-сферы, соответственно;

$R_{k i}$ - функция отклика (Response Function - RF) [16];

$k=1,2, \ldots, m-$ количество сфер, $m=11 ; i=1,2, \ldots, n$ - количество энергий;

В качестве начального спектра используется нормированный спектр $\left(\sum_{i=1}^{n} \varphi_{i}^{D S}=1\right) \operatorname{PuBe}$ источника нейтронов в диапазоне энергий $10^{-9} \leq E \leq 15$ (МэВ) [17].

Значения $n_{k C}$ и $n_{k}$ подчиняются $\chi^{2}$ - распределению со степенями свободы $m$ [15] с учетом того, что значения $n_{k}$ при измерении подчиняются распределению Пуассона и нормальному распределению.

Поэтому справедливы выражения

$$
\sum_{k=1}^{m}\left(\frac{\varepsilon_{k}}{\sigma_{k}}\right)^{2}=\chi^{2} \text { и } \sum_{k=1}^{m} \frac{\varepsilon_{k}}{\sigma_{k}}=0
$$

где:

суммарная стандартная неопределенность при измерении $n_{k}$ и расчете $n_{k C}$ : 


$$
\sigma_{k}=\sqrt{\sigma_{k A}^{2}+\sigma_{k B}^{2}}
$$

стандартная неопределенность типа А результатов измерений (среднее квадратическое отклонение - СКО) с учетом распределения Пуассона:

$$
\sigma_{k A}=\sqrt{N}
$$

$N$ - измеренное количество импульсов;

$\sigma_{k B}$ - стандартная неопределенность типа В при измерении $n_{k}$ (обусловлена неисключенной систематической погрешностью).

Измеренный спектр нейтронного излучения представим в виде $[11,12]$ :

$$
\varphi_{i}=\varphi_{i}^{D S} \cdot e^{-\sum_{k=1}^{m} R_{k i}\left(\lambda_{k}+\frac{\gamma}{\sigma_{k}}\right)}
$$

где $\lambda_{k}$ и $\gamma$ - параметры (множители Лагранжа), которые определены в $[11,12]$.

Аналитическое решение уравнения (3) с учетом (4), применяя теорему Байеса и метод множителей Лагранжа, приведено в $[13,15]$.

Расчет спектра нейтронного излучения выполнен с помощью подпрограммы MAXED for Few-Channel (FC) $(m<<n)-$ MXD_FC33 из программы UMG_3.3 $[11,12]$.

Алгоритм аналитического решения уравнения (3) с учетом (4), применяя матрицы и деконволюцию функций, приведен в $[11,12]$.

В данной работе косвенно измерены в диапазоне энергий $10^{-9} \leq E \leq 15$ (МэВ) следующие характеристики: $\varphi$, МАЭД $\dot{H}^{*}(10)$; коэффициент перехода от флюенса к АЭД $h_{\Phi}^{*}(10)$ и средняя энергия спектра $\bar{E}$, которые рассчитываются по формулам:

$$
\varphi=\sum_{i=1}^{n} \varphi_{i}, \quad \dot{H}^{*}(10)=\sum_{i=1}^{n} h_{\Phi i}^{*}(10) \cdot \varphi_{i}, \quad h_{\Phi}^{*}(10)=\frac{\dot{H}^{*}(10)}{\varphi}, \quad \bar{E}=\frac{\sum_{i=1}^{n} \varphi_{i} \cdot E_{l 0 i}}{\sum_{i=1}^{n} \varphi_{i}}
$$

где $\varphi_{i}=\varphi_{L i} \cdot u_{L i} ; \varphi_{L i}=\frac{\varphi_{i}}{u_{L i}} ; u_{L i}=\ln \frac{E_{i+1}}{E_{i}}-$ летаргия; $E_{l 0 i}=0,5 \cdot\left(E_{i+1}+E_{i}\right)-$ средняя энергия ячейки при линейном масштабе энергии.

Измеренные величины без конуса и с конусом рассчитываются по аналогичным формулам.

Диапазон энергий разбит на 5 поддиапазонов $(p)$ согласно радиационному взвешивающему фактору (коэффициенту качества) [18] (таблица 2).

Таблица 2.

Радиационный взвешивающий фактор

\begin{tabular}{|c|c|c|c|c|c|}
\hline Поддиапазон $(p)$ & $\begin{array}{c}1 \text { (тепловые } \\
\text { нейтроны) }\end{array}$ & 2 & 3 & 4 & 5 \\
\hline$E$, МэВ & $10^{-9} \leq E \leq 5 \cdot 10^{-7}$ & $5 \cdot 10^{-7}<E \leq 10^{-2}$ & $10^{-2}<E \leq 10^{-1}$ & $10^{-1}<E \leq 2$ & $2<E \leq 15$ \\
\hline$w_{R}$ & 5 & 10 & 20 & 10 \\
\hline
\end{tabular}

\section{РЕЗУЛЬТАТЫ ИССЛЕДОВАНИЙ}

C помощью спектрометра NEMUS на сферах Боннера проведены измерения АИС нейтронного излучения $\mathrm{PuBe}$ источника и АИС после прохождения нейтронного излучения через 6 образцов материалов без конуса и с конусом для 10 сфер и счетчика SP9. Всего измерено 154 АИС. Нормированный АИС нейтронного излучения, который постоянен и не зависит от энергии нейтронов [13], приведен на рис. 2.

Скорость счета импульсов вычислим по формуле

$$
n=\frac{N}{t_{L T}-N \cdot \tau}
$$

где $N=\sum_{j=65}^{600} N_{j}$ - суммарное количество импульсов АИС; $t_{L T}-$ разрешающее время AЦП FAST 7070 ; $\tau=16$ мкс - мертвое время счетчика SP9.

Стандартную неопределенность типа А определим как результат косвенного измерения

$$
\sigma_{k A}=\frac{1}{t_{L T}} \cdot \sqrt{\left(\sigma_{N_{\Sigma k}}\right)^{2}+\left(\sigma_{N_{K k}}\right)^{2}}
$$

где $\sigma_{N_{\Sigma k}}$ и $\sigma_{N_{K k}}$ вычислим по формуле (4.2). 


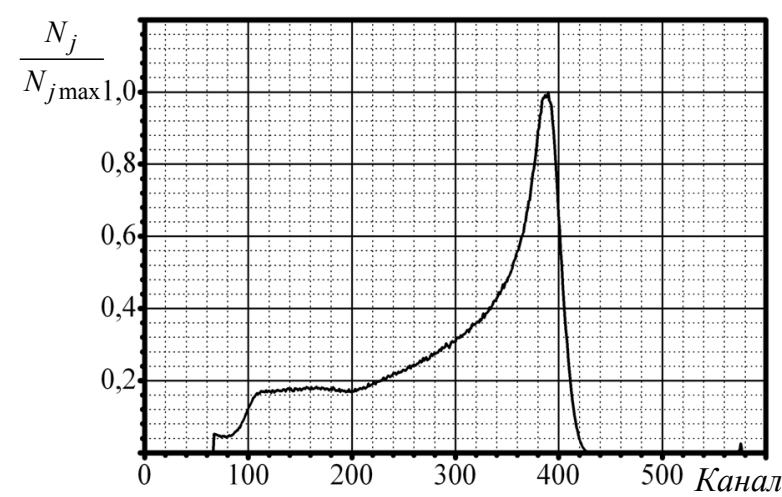

Рис. 2. Нормированный АИС нейтронного излучения $N_{j}$ - количество импульсов в ј-канале

При измерении спектров считаем, что относительная стандартная неопределенность типа В равна $\sigma_{\delta_{k B}}=0,05$.

Суммарную стандартную неопределенность $\sigma_{k}$ вычислим по формуле (4.1). Стандартная неопределенность типа А согласно распределению Пуассона определяется временем измерения количества импульсов.

Стандартная неопределенность типа В определяется погрешностью при расчете $R_{k i}$ [16]; правильностью расчета геометрических размеров конусов, качеством их изготовления, чистотой материала, из которого они изготовлены; размещением сферы относительно источника нейтронов; анизотропией счетчика SP9; нестабильностью AIOSAP-02 и АЦП FAST 7070.

По измеренным АИС построены зависимости $n_{\Sigma k}, n_{K k}, n_{k}=f(d)$ (рис. 3-5), знание которых позволило рассчитать спектры нейтронного излучения. По спектрам определено процентное соотношение нейтронов в заданных поддиапазонах энергий (таблица 2): $\frac{\varphi_{p}}{\varphi}, \frac{\varphi_{\Sigma p}}{\varphi_{\Sigma}}, \frac{\varphi_{K p}}{\varphi_{K}}=f(p)$ (рис. 6-8). Значения $n_{\Sigma k}$ и $n_{K k}$ рассчитывались по формуле (6); $n_{k}-$ по формуле (2); значения $\varphi, \dot{H}^{*}(10), h_{\Phi}^{*}(10)$ и $\bar{E}-$ по формулам (5).

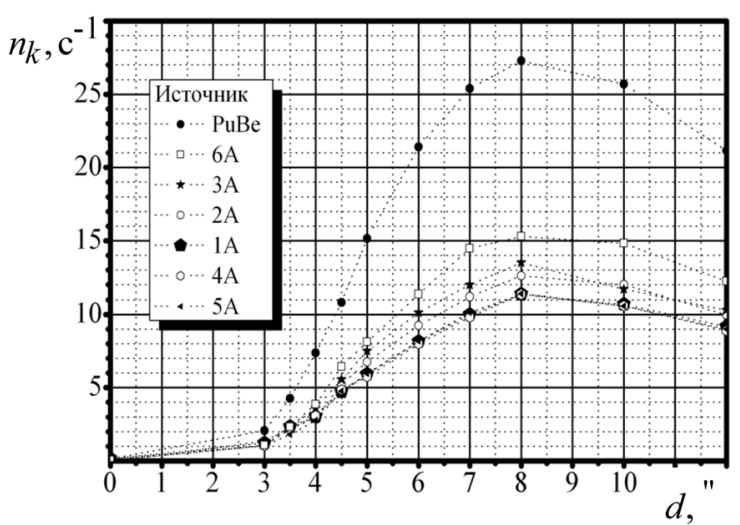

Рис. 3. Зависимости $n_{k}=f(d)$

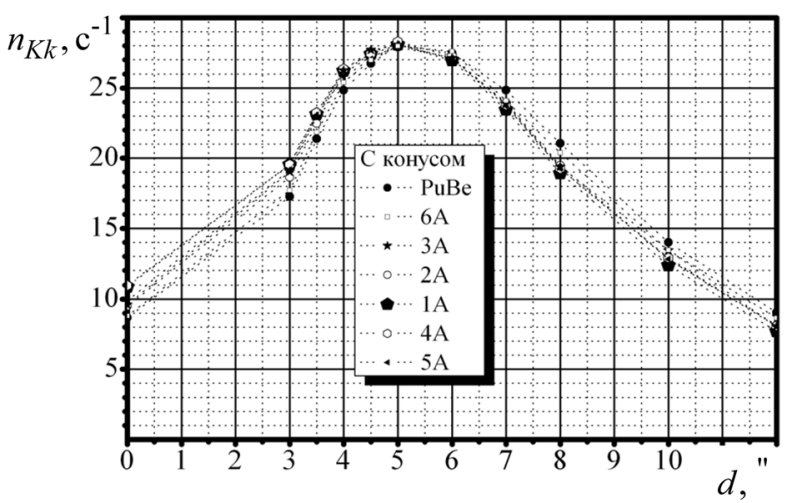

Рис. 5. Зависимости $n_{K k}=f(d)$

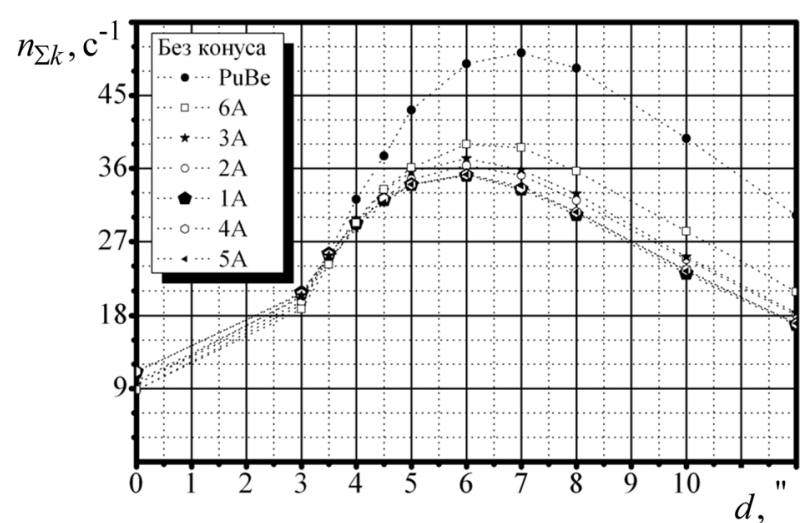

Рис. 4. Зависимости $n_{\Sigma k}=f(d)$

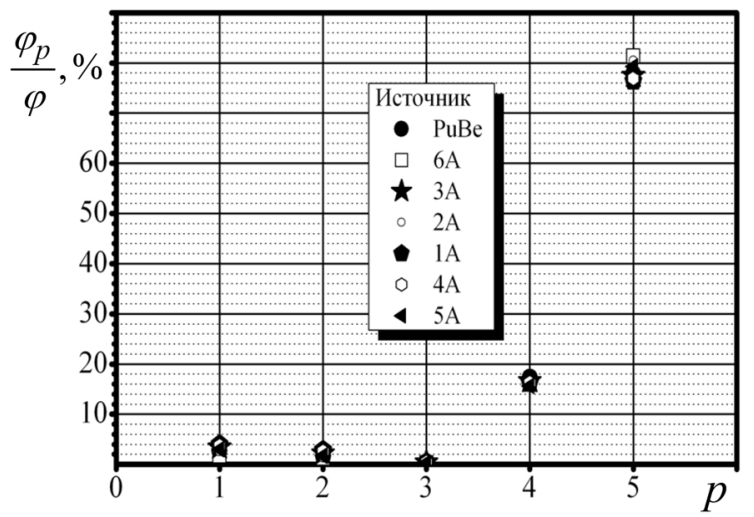

Рис. 6. Зависимости $\frac{\varphi_{p}}{\varphi}=f(p)$ 


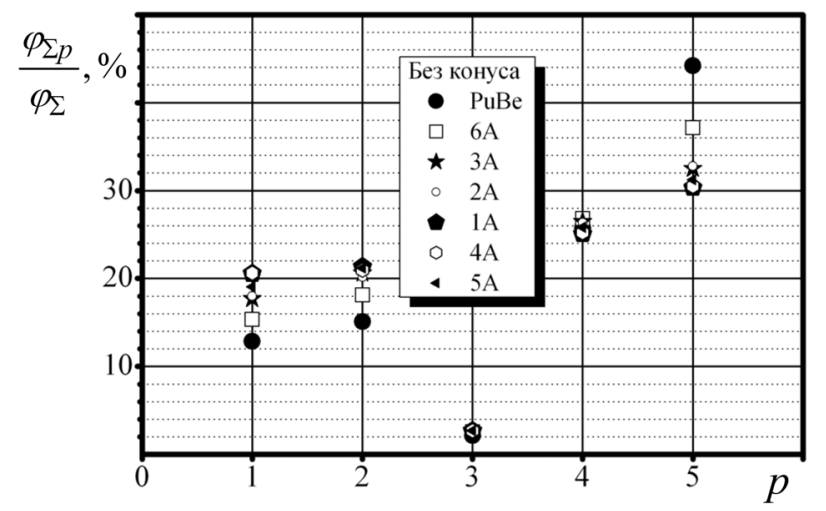

Рис. 7. Зависимости $\frac{\varphi_{\Sigma p}}{\varphi_{\Sigma}}=f(p)$

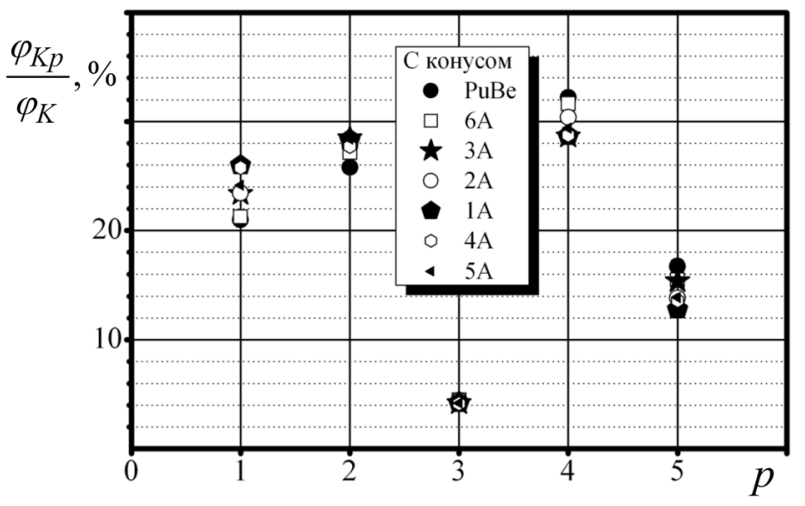

Рис. 8. Зависимости $\frac{\varphi_{K p}}{\varphi_{K}}=f(p)$

Нормированные спектры нейтронного излучения $\left(\sum_{i=1}^{n} \varphi_{i}=1 \mathrm{~cm}^{-2} \cdot \mathrm{c}^{-1}\right)($ ППН) без конуса приведены на рис. $9,10$.

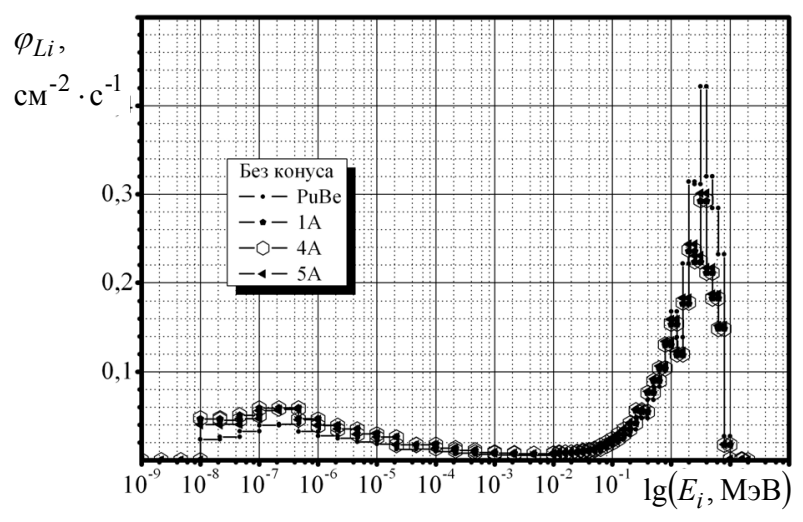

Рис. 9. Спектры нейтронного излучения (ППН) $\mathrm{PuBe}$ источника (образцы 1A, 4A и 5A)

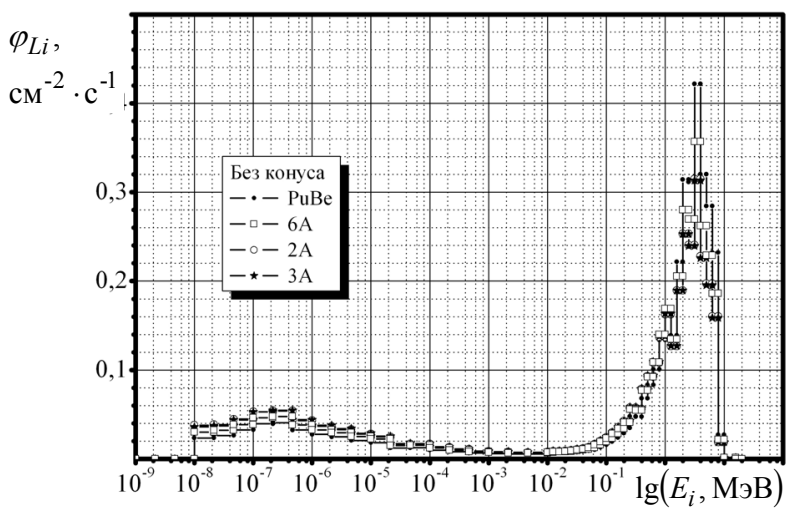

Рис. 10. Спектры нейтронного излучения (ППН) $\mathrm{PuBe}$ источника (образцы 2А, 3А и 6А)

Измеренные значения ППН, МАЭД, $\bar{E}_{\Sigma}$ и $h_{\Phi}^{*}(10)_{\Sigma}$; расширенные неопределенности при их измерении и значения ППН для поддиапазонов энергий приведены в таблицах $3-5$.

Значения ППН, МАЭД, $\bar{E}_{\Sigma}$ и $h_{\Phi}^{*}(10)_{\Sigma}$

\begin{tabular}{|c|c|c|c|c|c|c|c|c|}
\hline \multirow[t]{2}{*}{ Образец } & $\varphi$ & $\varphi_{\Sigma}$ & $\varphi_{K}$ & $\dot{H}^{*}(10)$ & $\dot{H}^{*}(10)_{\Sigma}$ & $\dot{H}^{*}(10)_{K}$ & \multirow{2}{*}{$\begin{array}{c}\bar{E}_{\Sigma}, \\
\text { МэВ }\end{array}$} & \multirow{2}{*}{$\begin{array}{l}h_{\Phi}^{*}(10)_{\Sigma}, \\
\Pi 3 \mathrm{~B} \cdot \mathrm{cm}^{2}\end{array}$} \\
\hline & \multicolumn{3}{|c|}{$\mathrm{cm}^{-2} \cdot \mathrm{c}^{-1}$} & \multicolumn{3}{|c|}{ мКЗВ ${ }^{-1}{ }^{-1}$} & & \\
\hline $\mathrm{PuBe}$ & 10,8 & 26,3 & 15,3 & 15,0 & 26,3 & 10,0 & 2,112 & 278 \\
\hline $6 \mathrm{~A}$ & 6,54 & 22,0 & 15,2 & 9,34 & 20,0 & 9,57 & 1,793 & 252 \\
\hline $3 \mathrm{~A}$ & 5,70 & 21,4 & 15,7 & 7,85 & 17,9 & 9,32 & 1,580 & 232 \\
\hline $2 \mathrm{~A}$ & 5,40 & 21,0 & 15,4 & 7,57 & 17,6 & 9,07 & 1,589 & 232 \\
\hline $1 \mathrm{~A}$ & 4,90 & 20,9 & 15,7 & 6,64 & 16,4 & 8,70 & 1,478 & 218 \\
\hline $4 \mathrm{~A}$ & 4,81 & 21,0 & 16,0 & 6,57 & 16,6 & 9,06 & 1,481 & 219 \\
\hline $5 \mathrm{~A}$ & 4,81 & 20,6 & 15,6 & 6,70 & 16,6 & 9,02 & 1,521 & 224 \\
\hline
\end{tabular}

Анализ зависимостей $n_{\Sigma k}, n_{K k}, n_{k}=f(d)$ (рис. 3-5), $\frac{\varphi_{p}}{\varphi}, \frac{\varphi_{\Sigma p}}{\varphi_{\Sigma}}, \frac{\varphi_{K p}}{\varphi_{K}}=f(p)$ (рис. 6-8), спектров нейтронного излучения (рис. 9,10) и данных, приведенных в таблице 3 , показывает следующее:

- для расчета линейного коэффициента ослабления необходимо измерить ППН без учета рассеянного нейтронного излучения без образца и с образцом;

- с точки зрения радиационной безопасности необходимо измерить МАЭД без конуса с образцом; 
- максимальное ослабление (минимальные значения $\varphi, \dot{H}^{*}(10)$ и $\left.\dot{H}^{*}(10)_{\Sigma}\right)$ получены для образцов 1А, 4А и 5А, минимальное ослабление - для образца 6А (рис. 3, таблица 3);

- зависимости $n_{k}=f(d)$ для образцов $1 \mathrm{~A}, 4 \mathrm{~A}$ и 5А в диапазоне $10^{-2}<E \leq 15$ (МэВ) совпадают (рис. 3), следовательно, совпадают и спектры ППН (рис. 6) [13]. В поддиапазоне $10^{-9} \leq E \leq 5 \cdot 10^{-7}$ (МэВ) (тепловые нейтроны) (рис. 6) для образца $5 \mathrm{~A}$ меньше нейтронов, что связано с наличием в этом образце изотопа ${ }^{10} B$;

- аналогично и для зависимостей $n_{\Sigma k}=f(d)$ для образцов 1А, 4A и 5А (рис. $\left.4,7,9\right)$;

- зависимости $n_{\Sigma k}=f(d)$ для образцов 2А и 3А практически совпадают (рис. 4), следовательно, практически совпадают и спектры ППН (рис. 7,10) [13];

- максимум зависимости $n_{\Sigma k}=f(d)$ РuBe источника нейтронов соответствует 7 сфере (рис. 4), для всех образцов - 6 сфере (рис. 4). Следовательно, спектры нейтронного излучения после прохождения нейтронов через образцы материалов смещены в область более низких энергий по сравнению со спектром РuВе источника нейтронов [13];

- средняя энергия спектра нейтронного излучения РuВе источника нейтронов для образцов 6А, 3А, 2A, 1А и $4 \mathrm{~A}$ уменьшается (таблица 3 ), соответственно, уменьшается и $h_{\Phi}^{*}(10)_{\Sigma}[13]$. Для образца 5А средняя энергия и $h_{\Phi}^{*}(10)_{\Sigma}$ больше, чем для образцов $1 \mathrm{~A} \mathrm{и} 4 \mathrm{~A}$, что связано с наличием в этом образце изотопа ${ }^{10} B$;

- максимум зависимости $n_{K k}=f(d)$ РuBe источника нейтронов и для всех образцов соответствует 5 сфере (рис. 5); это подтверждает тот факт, что с помощью конусов РuВе источник полностью затенен.

Таблица 4.

Расширенные неопределенности при измерении ППН, МАЭД, $\bar{E}_{\Sigma}$ и $h_{\Phi}^{*}(10)_{\Sigma}$.

\begin{tabular}{|c|c|c|c|c|c|c|c|c|}
\hline Образец & $U_{\delta}(\varphi)$ & $U_{\delta}\left(\varphi_{\Sigma}\right)$ & $U_{\delta}\left(\varphi_{K}\right)$ & $U_{\delta}\left(\dot{H}^{*}(10)\right)$ & $U_{\delta}\left(\dot{H}^{*}(10)_{\Sigma}\right)$ & $U_{\delta}\left(\dot{H}^{*}(10)_{K}\right)$ & $U_{\delta}\left(\bar{E}_{\Sigma}\right)$ & $U_{\delta}\left(h_{\Phi}^{*}(10)_{\Sigma}\right)$ \\
\hline $\begin{array}{c}\text { РuBe, } \\
\text { образцы }\end{array}$ & 0,04 & 0,04 & 0,04 & 0,04 & 0,05 & 0,06 & 0,09 & 0,05 \\
\hline
\end{tabular}

Примечание $-U_{\delta}(X)=k \cdot u_{\delta}(X)$ - расширенная относительная неопределенность при доверительной вероятности $P=0,95$ ( $k=2$ - коэффициент охвата); $u_{\delta}(X)$ - рассчитанные значения относительных суммарных стандартных неопределенностей.

Значения ППН для поддиапазонов энергий без учета рассеянного излучения, $\mathrm{cm}^{-2} \cdot \mathrm{c}^{-1}$.

\begin{tabular}{|c|c|c|c|c|c|c|}
\hline Образец & $\varphi_{1}$ & $\varphi_{2}$ & $\varphi_{3}$ & $\varphi_{4}$ & $\varphi_{5}$ & $\varphi$ \\
\hline \multirow{2}{*}{ PuBe } & 0,$294 ;$ & 0,$184 ;$ & 0,$043 ;$ & 1,$86 ;$ & 2,$39 ;$ & \\
& $S\left(E_{1}\right)=0,027$ & $S\left(E_{2}\right)=0,017$ & $S\left(E_{3}\right)=0,004$ & $S\left(E_{4}\right)=0,172$ & $S\left(E_{5}\right)=0,777$ & 10,8 \\
\hline 6A & 0,111 & 0,048 & 0,016 & 1,04 & 5,32 & 6,54 \\
\hline 3A & 0,194 & 0,125 & 0,023 & 0,946 & 4,41 & 5,70 \\
\hline 2A & 0,138 & 0,088 & 0,015 & 0,829 & 4,33 & 5,40 \\
\hline 1A & 0,198 & 0,142 & 0,022 & 0,793 & 3,74 & 4,90 \\
\hline 4A & 0,181 & 0,125 & 0,021 & 0,784 & 3,70 & 4,81 \\
\hline 5A & 0,146 & 0,083 & 0,016 & 0,752 & 3,81 & 4,81 \\
\hline
\end{tabular}

Примечание $-S\left(E_{p}\right)=\frac{\varphi_{p}}{\varphi}-$ доля нейтронов в поддиапазоне $p$.

Для расчета длины релаксации нейтронов $(L)$ и линейного коэффициента ослабления нейтронов в материале $(\Sigma)$ применим формулу, которая справедлива для немоноэнергетического источника нейтронов [5]:

$$
\varphi=\varphi_{0} \cdot \sum_{p=1}^{G} f_{p} \cdot e^{-\sum_{l=1}^{M} h_{l} / L_{p l}} \cdot S\left(E_{p}\right)=\sum_{p=1}^{G} f_{p} \cdot e^{-\sum_{l=1}^{M} h_{l} / L_{p l}} \cdot \varphi_{0 p},
$$

где $\varphi_{0}$ и $\varphi-$ ППН без образца и с образцом на расстоянии $\mathrm{R}$ от геометрического центра источника;

$l=1, \ldots, M ; M-$ количество толщин материала, для которых $L=$ Const;

$p=1, \ldots, G ; G$ - количество поддиапазонов энергий, для которых $p=$ Const;

$h_{l}$ - толщина $l$-го участка материала; 
$L_{p l}$ - длина свободного пробега нейтронов в материале или длина релаксации нейтронов в материале, которая характеризует экспоненциальную зависимость ослабления нейтронов $l$-го участка материала в поддиапазоне $p$ (ослабление ППН в $e \cong 2,7183$ раз);

$L_{p l}=\frac{1}{\Sigma_{p l}} ; \quad \Sigma_{p l}-$ линейный коэффициент ослабления нейтронов для $l$-го участка материала в поддиапазоне $p$;

$f_{p}$ - коэффициент, характеризующий отклонение от экспоненциальной формы кривой ослабления в образце для поддиапазона $p$ при $h=(2 \ldots 3) \cdot L_{p}$; если $L_{p}>h, f_{p}=1$.

В общем случае $L$ зависит от энергии нейтронов источника, толщины материала, компоновки и геометрии защиты, энергии детектируемых нейтронов [5].

Если $G=1$ и $M=1$, согласно (7)

$$
\varphi=\varphi_{0} \cdot e^{-h / L}=\varphi_{0} \cdot e^{-\Sigma \cdot h}
$$

откуда найдем

$$
L=\frac{1}{\Sigma}=\frac{h}{\ln \left(\frac{\varphi_{0}}{\varphi}\right)}
$$

Расширенную относительную неопределенность при доверительной вероятности $P=0,95$ при измерении $L$ с учетом того, что $U_{\delta}\left(\varphi_{0}\right)=U_{\delta}(\varphi)$ представим в виде

$$
U_{\delta}(L)=\sqrt{\left[U_{\delta}(h)\right]^{2}+2 \cdot\left[U_{\delta}(\varphi)\right]^{2} \cdot\left(\ln \frac{\varphi_{0}}{\varphi}\right)^{-2}},
$$

где $U_{\delta}(h)=0,05$ и $U_{\delta}(\varphi)=0,04$ - расширенные относительные неопределенности при доверительной вероятности $P=0,95$ при измерении толщины образца и ППН.

Длину релаксации нейтронов в массовых единицах представим в виде

$$
L_{m}=\rho \cdot L,
$$

где $\rho$ - плотность материала образца.

В данной работе рассмотрен случай, когда $f_{p}=1, G=5$ и $M=1$, поэтому согласно (7) и (8):

$$
\varphi=\varphi_{0} \cdot \sum_{p=1}^{5} e^{-h / L_{p}} \cdot S\left(E_{p}\right)=\sum_{p=1}^{5} e^{-h / L_{p}} \cdot \varphi_{0 p}=\varphi_{0} \cdot e^{-h / L_{\Sigma}}
$$

Выражение (12) представим в виде

$$
\sum_{p=1}^{5} \varphi_{p}=\sum_{p=1}^{5} e^{-h / L_{p}} \cdot \varphi_{0 p} .
$$

Суммарную длину релаксации нейтронов в материале определим из (12) как

$$
L_{\Sigma}=\left[\ln \sum_{p=1}^{5} e^{1 / L_{p}} \cdot S\left(E_{p}\right)\right]^{-1}
$$

Рассчитанные по данным таблиц 3-5 и формулам (9), (10), (13) и (14) с учетом (11) значения $\Sigma, L_{m}, U_{\delta}(L)$ и $L_{\Sigma m}$ приведены в таблице 6.

Таблица 6.

Характеристики материалов исследуемых образцов.

\begin{tabular}{|c|c|c|c|c|c|c|c|c|}
\hline Образец & $h, \mathrm{cм}$ & $\rho, г \mathrm{~cm}^{3}$ & $\Sigma, \mathrm{cм}^{-1}$ & $L, \mathrm{cм}$ & $\begin{array}{c}L_{m}, \Gamma^{*} \mathrm{cм}^{-2} \\
(9) \text { и (11) }\end{array}$ & $\begin{array}{c}L_{\Sigma m}, \Gamma^{*} \mathrm{cм}^{-2} \\
(14) \text { и (11) }\end{array}$ & $\delta L_{\Sigma m}, \%$ & $U_{\delta}(L), \%$ \\
\hline 6A & 6,40 & 1,36 & 0,078 & 12,8 & 17,4 & 17,8 & 2,3 & 12,5 \\
\hline 3A & 5,91 & 1,30 & 0,108 & 9,26 & 12,0 & 12,3 & 2,5 & 10,0 \\
\hline 2A & 6,25 & 1,23 & 0,111 & 9,01 & 11,1 & 11,4 & 2,7 & 10,0 \\
\hline 1A & 6,26 & 0,91 & 0,126 & 7,94 & 7,23 & 7,28 & 0,7 & 9,0 \\
\hline 4A & 6,35 & 0,92 & 0,127 & 7,87 & 7,24 & 7,39 & 2,1 & 9,0 \\
\hline 5A & 6,25 & 0,97 & 0,129 & 7,75 & 7,52 & 7,67 & 2,0 & 9,0 \\
\hline
\end{tabular}

Примечание - $\delta L_{\Sigma m}$ - отклонение от $L_{m}$.

Как видно из таблицы 6: 
- толщина образцов меньше длины релаксации нейтронов в материале образца, поэтому $f_{p}=1$;

- максимальный линейный коэффициент ослабления нейтронов получен для образцов 1А, 4A и 5А, минимальный - для образца 6 А, что соответствует зависимостям $n_{k}=f(d)$ (рис. 3 );

- значения длины релаксации в массовых единицах, рассчитанные по формулам (9), (11) и (14), (11), находятся в пределах расширенных неопределенностей при $P=0,95$.

Следует отметить, что при исследовании защиты от нейтронного излучения с точки зрения радиационной безопасности необходимо измерять мощность поглощенной дозы (МПД) в воздухе [19].

МАЭД нейтронного излучения равна [18]:

$$
\dot{H}^{*}(10)=\sum_{p=1}^{5} w_{R p} \cdot \dot{D}_{p}
$$

где $\dot{D}_{p}-$ МПД в воздухе нейтронного излучения;

$w_{R p}$ и $p$ приведены в таблице 2.

Следовательно, МПД в воздухе нейтронного излучения без конуса (таблица 7) рассчитаем по формуле

$$
\dot{D}=\frac{\dot{H}^{*}(10)_{1}+\dot{H}^{*}(10)_{2}}{5}+\frac{\dot{H}^{*}(10)_{3}}{10}+\frac{\dot{H}^{*}(10)_{4}}{20}+\frac{\dot{H}^{*}(10)_{5}}{10}
$$

где $\dot{H}^{*}(10)_{p}$ приведены в таблице 7.

Таблица 7.

\begin{tabular}{|c|c|c|c|c|c|c|c|}
\hline Образец & $\begin{array}{c}\dot{H}^{*}(10)_{\Sigma 1}, \\
\text { мКЗВ } \cdot{ }^{-1}\end{array}$ & $\begin{array}{c}\dot{H}^{*}(10)_{\Sigma 2}, \\
\text { мкЗв }{ }^{-ч^{-1}}\end{array}$ & $\begin{array}{c}\dot{H}^{*}(10)_{\Sigma 3}, \\
\text { мКЗВ } \cdot{ }^{-1}\end{array}$ & $\begin{array}{c}\dot{H}^{*}(10)_{\Sigma 4}, \\
\text { мкЗв }{ }^{-1}{ }^{-1}\end{array}$ & $\begin{array}{c}\dot{H}^{*}(10)_{\Sigma 5} \\
\text { мкЗв }{ }^{-1}\end{array}$ & $\begin{array}{l}\dot{H}^{*}(10)_{\Sigma}, \\
\text { мкЗв }{ }^{-1}\end{array}$ & $\begin{array}{c}\dot{D}_{\Sigma}, \\
\mathrm{M \kappa} \Gamma \mathrm{p} \cdot \mathrm{ч}^{-1}\end{array}$ \\
\hline $\mathrm{PuBe}$ & 0,15 & 0,16 & 0,09 & 11,6 & 14,3 & 26,3 & 2,1 \\
\hline $6 \mathrm{~A}$ & 0,15 & 0,16 & 0,12 & 9,61 & 9,96 & 20,0 & 1,6 \\
\hline $3 \mathrm{~A}$ & 0,17 & 0,18 & 0,12 & 8,97 & 8,41 & 17,9 & 1,4 \\
\hline $2 \mathrm{~A}$ & 0,17 & 0,17 & 0,15 & 8,78 & 8,33 & 17,6 & 1,4 \\
\hline $1 \mathrm{~A}$ & 0,19 & 0,18 & 0,14 & 8,23 & 7,66 & 16,4 & 1,3 \\
\hline $4 \mathrm{~A}$ & 0,19 & 0,18 & 0,16 & 8,34 & 7,73 & 16,6 & 1,3 \\
\hline $5 \mathrm{~A}$ & 0,17 & 0,18 & 0,16 & 8,37 & 7,76 & 16,6 & 1,3 \\
\hline
\end{tabular}

Значения МАЭД и МПД в воздухе без конуса

Примечание $-U_{\delta}\left(\dot{H}^{*}(10)_{\Sigma}\right)=U_{\delta}\left(\dot{D}_{\Sigma}\right)=0,05$.

Как видно из таблицы 7, минимальные значения МПД в воздухе нейтронного излучения получены для образцов $1 \mathrm{~A}, 4 \mathrm{~A}$ и $5 \mathrm{~A}$.

Нормированные спектры нейтронного излучения $\left(\sum_{i=1}^{n} \varphi_{i}=1 \mathrm{~cm}^{-2} \cdot \mathrm{c}^{-1}\right)$ (МАЭД) без конуса приведены на рис. 11 , зависимости $\frac{\dot{H}_{\Sigma p}^{*}}{\dot{H}_{\Sigma}^{*}}=f(p)$ - на рис. 12. Так как спектры ППН для образцов 1А, 4А и 5А практически совпадают, аналогично и для образцов 2А и $3 \mathrm{~A} \mathrm{(рис.} \mathrm{4,7),} \mathrm{то} \mathrm{на} \mathrm{рис.} 11$ приведены спектры МАЭД для РиВе источника, образцов $3 \mathrm{~A}, 4 \mathrm{~A}$ и $6 \mathrm{~A}$.

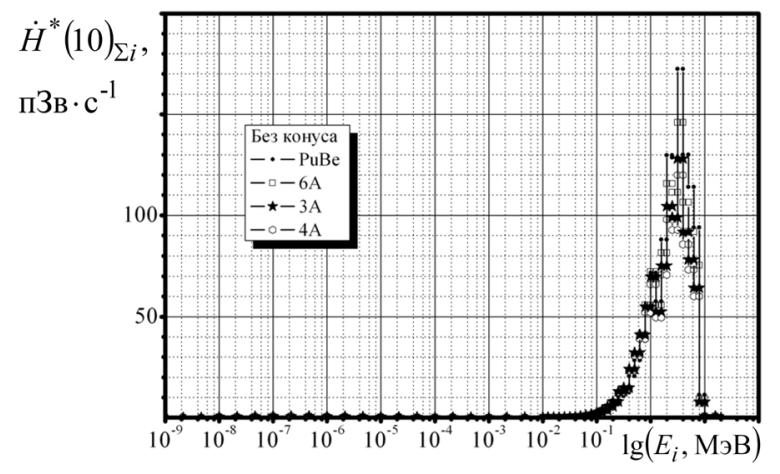

Рис. 11. Спектры нейтронного излучения (МАЭД) PuBe источника (образцы $3 \mathrm{~A}, 4 \mathrm{~A}$ и $6 \mathrm{~A}$ )

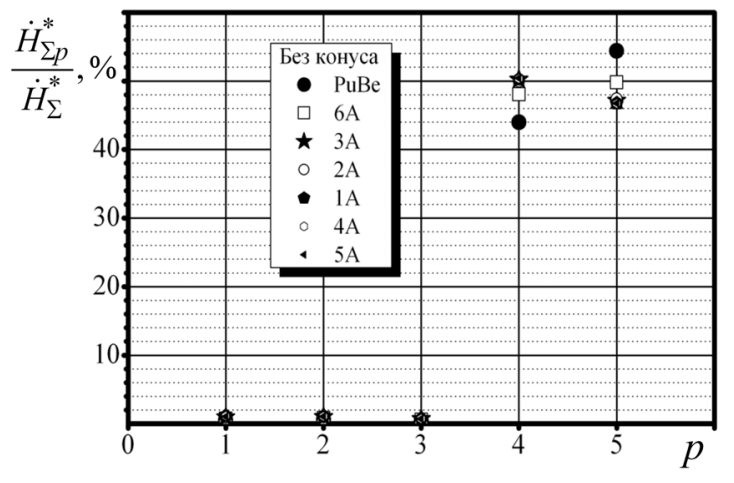

Рис. 12. Зависимости $\frac{\dot{H}_{\Sigma p}^{*}}{\dot{H}_{\Sigma}^{*}}=f(p)$ 
Согласно рис. 11,12 МАЭД для образцов 4А (1А и 5А) меньше, чем для образцов 3А (2A), 6A и РuBe источника.

В таблице 8 приведены значения $\Sigma$ и $L_{m}$ при $f=1$ для ПВД (таблица 6) и полученные по данным компании KOPOS KOLIN a.s. (Чехия) - производителя нейтростопов из ПВД и ПВД+В [20].

Таблица 8.

Значения $\Sigma$ и $L_{m}$ при $f=1$ для ПВД

\begin{tabular}{|c|c|c|c|}
\hline$h, \mathrm{~cm}$ & 6,35 & $44[20]$ & $90[20]$ \\
\hline$Q, \mathrm{pa} 3$ & 2,245 & $100[20]$ & $1000[20]$ \\
\hline$\Sigma, \mathrm{cM}^{-1}$ & 0,127 & 0,105 & 0,077 \\
\hline$L_{m}, \Gamma^{\cdot} \mathrm{cM}^{-2}$ & 7,27 & 8,80 & 12,0 \\
\hline
\end{tabular}

По данным таблицы 8 получена эмпирическая формула для оценки $\Sigma$ при проектировании защиты от нейтронного излучения в зависимости от толщины ПВД при использовании РuВе источника нейтронов:

где числовое значения $\Sigma(h)$ выражено в см$^{-1}, h-$ в см.

$$
\Sigma(h)=0,131-6 \cdot 10^{-4} \cdot h,
$$

Погрешность аппроксимации формулы (15) не превышает $\pm 0,5 \%$.

Так как значения $\Sigma$ для парафина, ПВД и ПВД+В приблизительно одинаковы (таблица 6), то формулой (15) можно пользоваться для оценки защитных свойств данных материалов от нейтронного излучения.

Окончательно отметим, что при выборе материала при проектировании защиты от нейтронного излучения необходимо учитывать следующее:

- максимальный линейный коэффициент ослабления нейтронов в материале;

- условия эксплуатации (температура);

- технологический процесс изготовления (простота);

- стоимость.

\section{ВЫВОДЫ}

1. По результатам исследований установлено следующее:

- для расчета линейного коэффициента ослабления необходимо измерить ППН без учета рассеянного нейтронного излучения без образца и с образцом;

- с точки зрения радиационной безопасности необходимо измерить МАЭД или МПД в воздухе с учетом рассеянного нейтронного излучения с образцом;

- максимальный линейный коэффициент ослабления нейтронов получен для ПВД+В, ПВД и парафина, минимальный - для карбида бора; следовательно, для защиты от источников нейтронов лучше применять ПВД+В, ПВД или парафин; для защиты от тепловых нейтронов лучше применять ПВД+В;

- спектры нейтронного излучения (ППН) после прохождения нейтронов через образцы материалов смещены в область более низких энергий по сравнению со спектром РuBe источника нейтронов.

2. Получена эмпирическая формула для оценки линейного коэффициента ослабления нейтронов при проектировании защиты от источников нейтронов в зависимости от толщины парафина, ПВД или ПВД+В.

\section{СПИСОК ЛИТЕРАТУРЫ}

1. Wiegel B., Alevra A.V. NEMUS - the PTB Neutron Multisphere Spectrometer: Bonner sphere and more // Nuclear Instruments and Method in Physics Research. Section A. - 2002. - Vol. 476. - P. 36-41.

2. Reginatto M., Goldhagen P., Neumann S. Spectrum unfolding, sensitivity analysis and propagation of uncertainties with the maximum entropy deconvolution code MAXED // Nuclear Instruments and Method in Physics Research. Section A. - 2002. Vol. 476. - P. 242-246.

3. Reginatto M., Goldhagen P. MAXED, A computer code for maximum entropy deconvolution of multisphere neutron spectrometer data // Health Physics. - 1999. - Vol. 77. - No. 5. - P. 579-583.

4. Reginatto M. What can we learn about the spectrum of high-energy stray neutron fields from Bonner sphere measurements? // Radiation Measurements. - 2009. - Vol. 44. - P. 692-699.

5. Mashkovich V.P., Kudryavceva A.V. Protection against ionizing radiation: Handbook. - M.: Energoatomizdat, $1995 .-496$ p. (in Russian)

6. $\quad$ Kozlov V.F. Handbook of Radiation Safety. - M.: Energoatomizdat, 1987. - 192 p. (in Russian)

7. Packing unit for transportation of radioisotope sources of neutrons: Patent 98578 Ukraine, MKI G21F 5/00 / V.E. Ostrovskykh, G.V. Sapelkina (Ukraine); Zayavleno 23.12.2014; Publ. 27.04.2015. - 4 p. (in Ukrainian)

8. Radiation-protective packaging device for transportation of radioisotope sources of neutrons: Patent 107486 Ukraine, MKI G21F 5/00 / V.E. Ostrovskykh, E.V. Rudichev, G.V. Sapelkina (Ukraine); Zayavleno 03.12.2015; Publ. 10.06.2016. - 4 p. (in Ukrainian)

9. WebMATE. User Manual. ICx Technologies GmbH. WebMATE/en/1.4(3967). - Solingen. - 2009. - 16 p.

10. WinTMCA32. User Manua. ICx Technologies GmbH. WebMATE/en/1.2(3366). - Solingen. - 2009. - 134 p.

11. Reginatto M., Wiegel B., Zimbal A., UMG 3.3 - Unfolding with MAXED and GRAVEL, available from the NEA Data Bank // http://www.oecd-nea.org/tools/abstract/detail/nea-1665, https://rsicc.ornl.gov/codes/psr/psr5/psr-529.html.

12. Reginatto M. The «few-channel» unfolding programs in the UMG package: MXD_FC33, GRV_FC33 and IQU_FC33. UMG package, version 3.3. PTB. - Braunschweig. -2004 . -51 p. 
13. Ivanskyi V.B., Letuchyi A.N., Orobinskyi A.N., Siroko H.V. Measurement of the main characteristics of PuBe and ${ }^{238} \mathrm{PuBe}$ neutron sources using Bonner sphere spectrometer with a ${ }^{3}$ He-counter // East European Journal of Physics. V.N. Karazin Kharkiv National University, Publishing. - 2017. - Vol. 4. - No 2. - P. 53-65. (in Russian)

14. Ivanskyi V.B., Letuchyi A.N., Orobinskyi A.N., Siroko H.V. Researches of the spectrum of PuBe and ${ }^{238} \mathrm{PuBe}$ neutron sources using Bonner sphere spectrometer with a ${ }^{3} \mathrm{He}$-counter // Proceedings of the International scientific-technical conference «METROLOGY-2017». - Minsk. - 2017. - P. 136 - 141. (in Russian)

15. Reginatto M., Goldhagen P. MAXED. A computer code for the deconvolution of multisphere neutron spectrometer data using the maximum entropy method // Environmental Measurements Laboratory. EML-595. U.S. Department of Energy. - New York. $-1998 .-40$ p.

16. Wiegel B., Alevra A.V., Siebert B.R.L. Calculations of the Response Functions of Bonner Spheres with a Spherical 3He Proportional Counter. Using a Realistic Detector Model / PTB-Bericht N-21. - Braunschweig. - 1994. - 84 p.

17. Compendium of Neutron Spectra and Detector Responses for Radiation Protection Purposes. Supplement to Technical Reports Series No. 318 / Technical Reports Series No. 403. IAEA. - Vienna. - 2001. - 337 p.

18. Compendium of Dose Coefficients based on ICRP Publication 60 / ICRP Publication 119. - International Commission on Radiological Protection. - 2012. - 130 p.

19. Radiation Hygiene. Basic sanitary regulations of radiation safety of Ukraine. State sanitary regulations. 6.177-2005-09-02. Kyiv. - Vidannya oficiine. - 74 p. (in Ukrainian)

20. Shielding bricks NEUTROSTOP // http://www.kopos.com/en/neu-neutrostop. 